\title{
The chromatin-remodeling protein ATRX is critical for neuronal survival during corticogenesis
}

\author{
Nathalie G. Bérubé,, ${ }^{1}$ Marie Mangelsdorf, ${ }^{1}$ Magdalena Jagla, ${ }^{1}$ Jackie Vanderluit, ${ }^{2}$ David Garrick, ${ }^{3}$ \\ Richard J. Gibbons, ${ }^{3}$ Douglas R. Higgs, ${ }^{3}$ Ruth S. Slack, ${ }^{2}$ and David J. Picketts ${ }^{1,4}$ \\ ${ }^{1}$ Molecular Medicine and ${ }^{2}$ Neuroscience Programs, Ottawa Health Research Institute, Ottawa, Ontario, Canada. \\ 3 Weatherall Institute of Molecular Medicine, John Radcliffe Hospital, Oxford, United Kingdom. \\ ${ }^{4}$ Departments of Medicine and Biochemistry, Microbiology, and Immunology, University of Ottawa, Ottawa, Ontario, Canada.
}

\begin{abstract}
Mutations in genes encoding chromatin-remodeling proteins, such as the $A T R X$ gene, underlie a number of genetic disorders including several $X$-linked mental retardation syndromes; however, the role of these proteins in normal CNS development is unknown. Here, we used a conditional gene-targeting approach to inactivate Atrx, specifically in the forebrain of mice. Loss of ATRX protein caused widespread hypocellularity in the neocortex and hippocampus and a pronounced reduction in forebrain size. Neuronal "birthdating" confirmed that fewer neurons reached the superficial cortical layers, despite normal progenitor cell proliferation. The loss of cortical mass resulted from a 12-fold increase in neuronal apoptosis during early stages of corticogenesis in the mutant animals. Moreover, cortical progenitors isolated from Atrx-null mice undergo enhanced apoptosis upon differentiation. Taken together, our results indicate that ATRX is a critical mediator of cell survival during early neuronal differentiation. Thus, increased neuronal loss may contribute to the severe mental retardation observed in human patients.
\end{abstract}

\section{Introduction}

Corticogenesis requires the establishment of a sufficient pool of neuroprogenitors that eventually undergo temporally coordinated waves of mitotic exit, migration, and terminal differentiation to generate the 6 layers of the mammalian cortex (1). Accordingly, differentiation is known to depend on genetic and epigenetic mechanisms that coordinate the repertoire of expressed genes that are vital for this developmental progression. Although several transcription factors have been shown to be essential for cortical organization (2-4), the function of chromatin-remodeling proteins in this process remains poorly defined. The importance of epigenetic factors in neurodevelopment is indicated by the finding that mutations in genes encoding chromatin-remodeling proteins are the cause of X-linked mental retardation (XLMR) disorders (5). This paradigm was established by the identification of the ATRX gene as the cause of the $\alpha$-thalassemia mental retardation, X-linked (ATR-X) syndrome (6) and the subsequent identification of mutations in the $M e C P 2$ and RPS6KA3 genes as the causes of Rett syndrome and Coffin-Lowry syndrome, respectively $(7,8)$.

Individuals affected with the ATR-X syndrome display moderate to severe cognitive delay associated with $\alpha$-thalassemia, facial dysmorphism, microcephaly, skeletal and genital abnormalities, and severe neonatal hypotonia (9). ATRX gene mutations at Xq13

Nonstandard abbreviations used: ATRX or ATR-X, $\alpha$-thalassemia mental retardation, X-linked; DAXX, Fas death domain-associated protein; Dnmt3, DNA methyltransferase 3 ; HP1 $\alpha$, heterochromatin protein $1 \alpha$; MAP2, microtubule-associated protein $2 ; n e o^{\mathrm{r}}$, neomycin-resistance gene; PHD, plant homeodomain; PML, promyelocytic leukemia; SNF2, sucrose-nonfermenting 2; SWI, mating-type switching; XLMR, $\mathrm{X}$-linked mental retardation.

Conflict of interest: The authors have declared that no conflict of interest exists.

Citation for this article: J. Clin. Invest. 115:258-267 (2005)

doi:10.1172/JCI200522329 are the underlying genetic cause of this syndrome $(6,10)$ and a growing list of other XLMR syndromes with overlapping developmental defects (11-14) or mild XLMR $(15,16)$.

The full-length ATRX protein contains a plant homeodomaintype (PHD-type) zinc finger motif and a DNA-dependent ATPase domain of the sucrose-nonfermenting 2 (SNF2) family, which suggests that it is a chromatin-remodeling protein $(17,18)$. These 2 domains are highly conserved between mouse and human (19) and correspond to the major mutation sites in affected males $(10,20)$ which suggests that they are also essential for normal function. A second smaller protein encoded by the ATRX gene, ATRXt, consists of the first 11 exons and retains the PHD zinc finger domain but not the SNF2 domain (21). Interestingly, mutations that affect both isoforms are no more severe than mutations that alter only the full-length protein (21).

Although primary structure suggests a role in chromatin regulation for ATRX, it is not clear whether ATRX imparts predominantly a positive or repressive effect on chromatin. ATRX localizes to pericentromeric heterochromatin and associates with heterochromatin protein $1 \alpha(\mathrm{HP} 1 \alpha)$, an adaptor molecule involved in the propagation of heterochromatic domains $(22,23)$. Recent biochemical characterization of an ATRX-containing complex has demonstrated that ATRX interacts with Fas death domain-associated protein (DAXX) in promyelocytic leukemia (PML) nuclear bodies $(24,25)$. Moreover, this complex has ATP-dependent remodeling activity (24) that is attenuated by mutations found in patients with ATRX (25). ATRXt has also been shown to colocalize with ATRX and HP1 proteins at pericentromeric heterochromatin, but not with DAXX and PML, which thereby suggests a distinct role for the C-terminal half of the molecule with DAXX (21). A repressive role is also suggested by homology to the DNA methyltransferase 3 (Dnmt3) family in the zinc finger region (26) and the observation of abnormal methylation patterns at several genomic loci in ATR-X patients (27). 

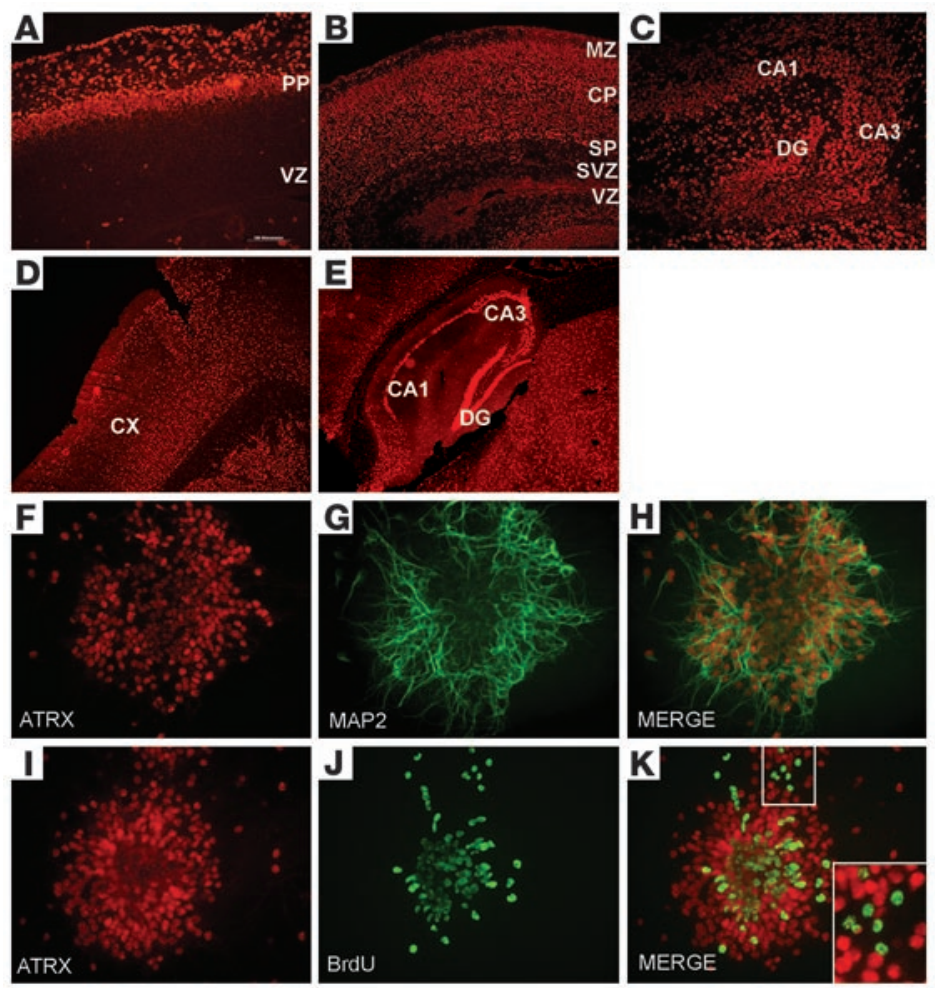

\section{Figure 1}

ATRX expression increases in postmitotic neurons. (A-E) Fixed tissue sections were treated with a polyclonal antibody $(\mathrm{H} 300)$ detecting the full-length ATRX protein. (A) Coronal section through the cortical region at E13.5 reveals highest expression in the preplate (PP). (B) Sagittal section through the cortex at P0.5 shows high levels of ATRX staining in postmitotic neurons of the marginal zone (MZ), cortical plate (CP), and subplate (SP), but lower levels of staining in the proliferative subventricular (SVZ) and ventricular zones (VZ). (C) Sagittal section at P0.5 through the hippocampus demonstrates high ATRX levels in CA1, CA3, and dentate gyrus (DG) subregions. (D and E) Saggital section at P31 through the brain demonstrates that ATRX expression is maintained in the adult throughout the cortex (CX), the hippocampus (CA1 and CA3), and the dentate gyrus subregions. (F-K) Cortical progenitor cells dissected from E12.5 telencephaIon were cultured for 6 days and were costained for ATRX (F) and for MAP2 $(\mathbf{G})$, a marker of neuronal differentiation. The merged image $(\mathbf{H})$ reveals coexpression of ATRX and MAP2 in the differentiated cells. In contrast, progenitors costained for ATRX (I) and proliferating cells as measured by BrdU incorporation $(\mathbf{J})$ show low levels of ATRX in the BrdU-positive cells (K, merged image). Higher magnification $(\times 20, \mathbf{K}$, bottom-right inset) image shows that BrdU and ATRX staining does not significantly overlap. Magnification, $\times 20(\mathbf{A}, \mathbf{C}-\mathbf{K}), \times 10(\mathbf{B})$.
Despite all these links to repression, a role for ATRX in gene activation is demonstrated by the reduced expression of $\alpha$-globin in many ATR-X patients with a mild form of $\alpha$-thalassemia (9) and the identification of somatic mutations in the ATRX gene in patients with $\alpha$-thalassemia-associated myelodysplasia (28). Thus, a "snapshot" of ATRX function has emerged as a regulator of chromatin accessibility at numerous loci including the $\alpha$-globin cluster and unknown target genes that are crucial for development.

\section{Figure 2}

Generation of Atrx gene-targeted mice. (A) A 6.2-kb Sstl fragment of the Atrx gene was used to engineer a targeting construct containing a floxed $\mathrm{NeO}^{\mathrm{r}}$ cassette within intron 17 and loxP sites flanking exon 18. Two herpes simplex virus thymidine kinase (HSV TK) genes were included to allow for positive and negative selection of recombinant ES cell clones. Female mice homozygous for the floxed Atrx allele (Atrx ${ }^{\text {loxP }}$ ) were bred to heterozygous Foxg1Cre male mice to generate $\mathrm{KO}$ male mice, referred to as AtrxFoxg1Cre. (B) Southern blot analysis of genomic DNA isolated from the cortices of newborn pups of different genotypes. $\mathrm{F} / \mathrm{Y}$, floxed-ATRX X chromosome; F/WT, wild-type $\mathrm{X}$ chromosome; $\mathrm{Cre}^{+}$, Cre-positive; Cre ${ }^{-}$, Cre-negative. Right margin: F, floxed allele; $R$, recombined allele. (C) RT-PCR analysis of RNA isolated from newborn forebrains of Atrx Foxg1Cre males (F/Y, Cre +), control littermates (F/Y, Cre -), or heterozygous females (F/WT) with or without Cre. Fragments were amplified with Atrx primers $17 \mathrm{~F}$ and $20 \mathrm{R}$, as indicated in A, or Gapdh primers (lower band) in control reactions. As expected, the amplified product was shorter when Cre was present due to recombination of loxP sites. (D) Western blot analysis of proteins isolated from newborn forebrains of wild-type, heterozygous, or knockout mice using an antibody that recognizes both full-length ATRX protein and the truncated isoform ATRXt (39f) or a C-terminal antibody (H300) that detects only the full-length isoform. Loss of full-length ATRX expression is observed in knockout animals, while ATRXt expression is not affected. Tubulin was used as a loading control.

To address the role of the full-length ATRX protein in CNS development, we generated a conditional Atrx knockout mouse using the Cre-loxP recombination system controlled by the Forkhead box G1 (Foxg1; ref. 29) and Nestin promoters, which confine Cre recombinase expression to either the forebrain or CNS neuroprogenitors, respectively. We show that loss of ATRX during early
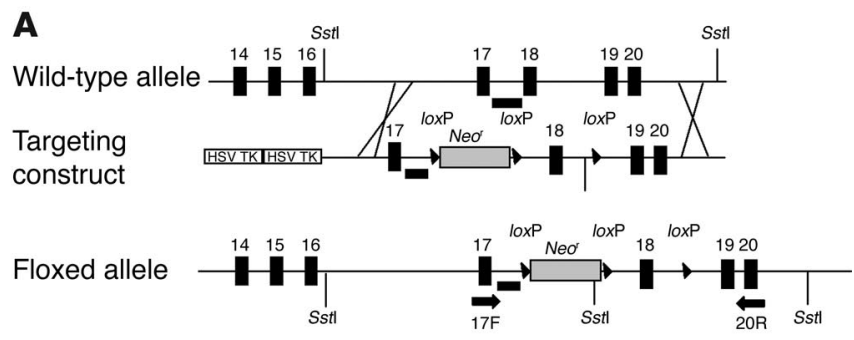

B

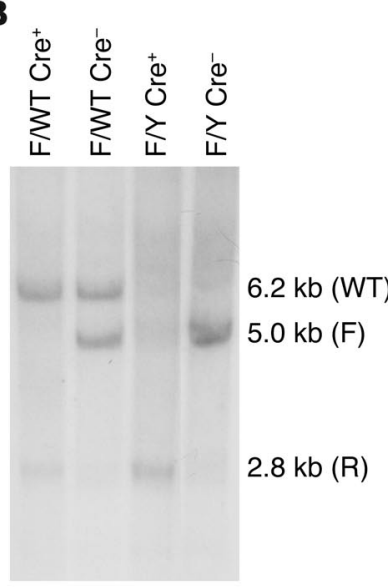

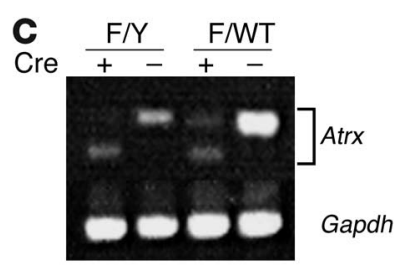

D

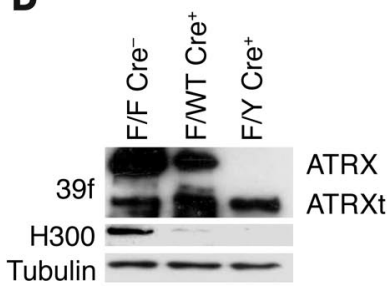



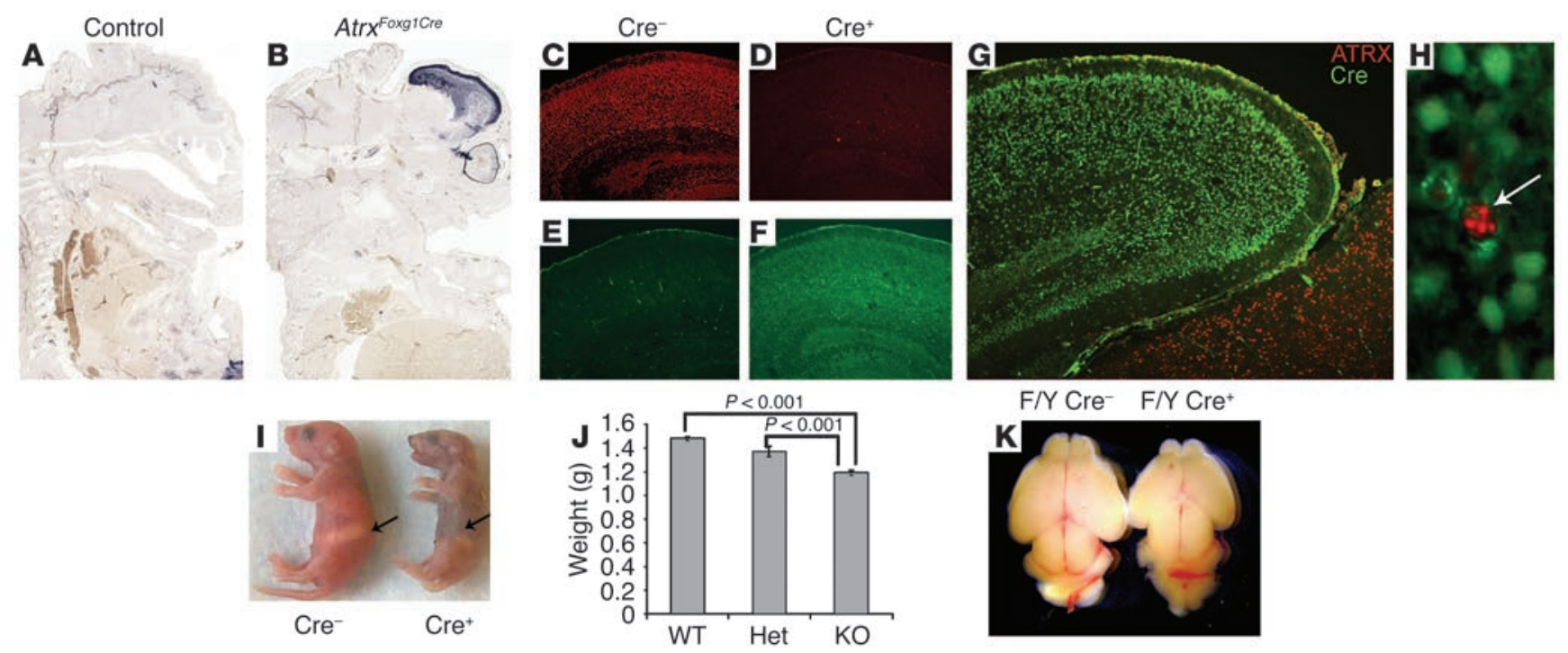

\section{Figure 3}

Cre-mediated conditional deletion of Atrx in the forebrain. (A and B) Cre in situ detection in sagittal sections of control (A) and Atrx Foxg1Cre (B) E13.5 embryos demonstrates that Cre expression is restricted to the telencephalon and anterior retina. (C-F) Sections of the cortex at P0.5 immunostained with antibodies specific for ATRX (C and $\mathbf{D})$ and Cre recombinase (E and $\mathbf{F})$. ATRX protein is undetectable in AtrxFoxg1Cre male cortex, and loss of expression is correlated with the presence of Cre recombinase. (G and $\mathbf{H}$ ) Merged image of ATRX expression (red) and Cre expression (green) demonstrating the loss of ATRX protein in the cortex but not in neighboring brain tissues and the presence of rare ATRX-positive cells in the cortex (arrow, H). (H) Higher magnification of the image in G. (I) Reduced size of P0.5 AtrxFoxg1Cre pup and lack of milk in stomach (indicated by arrows). (J) Graph depicting decreased weight of P0.5 AtrxFoxg1Cre males compared with that of wild-type and heterozygous Cre ${ }^{+}$ female mice $(P<0.001)$. (K) Reduced size of AtrxFoxg1Cre forebrain compared with that of a littermate control, with greater reduction in caudalmedial area. Magnification, $\times 10(\mathbf{C}-\mathbf{G})$ and $\times 40(\mathbf{H})$.

embryogenesis leads to significant increases in cortical progenitor cell apoptosis, whereas the proliferative capacity remains largely unchanged. As a result, the neonatal forebrain is abnormal with a major reduction in the caudal-medial cortex, loss of the dentate gyrus, and hypocellularity of several cortical layers. We propose that transcription-dependent events regulated by ATRX play a critical role in mediating the survival of neurons in the developing cortex and hippocampus.

\section{Results}

ATRX protein levels increase upon neuronal differentiation. The normal pattern of ATRX expression in the developing cortex was examined by immunostaining with an ATRX-specific antibody that detects the full-length isoform (22). ATRX staining was nuclear in all cells and coincided with regions of intense DAPI staining, consistent with the heterochromatin colocalization previously reported in murine cells (Figure 1, A-C; ref. 23). ATRX expression was detected as early as E11.5 in the telencephalic neuroepithelium, although at low levels (data not shown). At E13.5, postmitotic cells of the preplate expressed higher levels of ATRX protein than did neuroprogenitors of the ventricular zone (Figure 1A). In newborn pups (P0.5-P1.5) the full-length protein was expressed throughout the cortex (Figure 1B) and hippocampus (Figure 1C) but with the strongest signal confined to the postmitotic cells. Moreover, this signal was maintained in adult animals (Figure 1, D and E; ref. 30). Thus, the pattern of ATRX expression follows the process of neuroprogenitor differentiation. To define this pattern of expression more precisely, we examined ATRX expression levels in primary cortical cells derived from E12.5 telencephalon. After 6 days in culture, the majority of cells had established features of the differentiated state, including the expression of microtubuleassociated protein 2 (MAP2), a marker of neuronal differentiation (Figure 1G). Cells stained with MAP2 expressed high levels of the ATRX protein (Figure 1, F and $\mathrm{H}$ ), confirming the pattern of expression observed in the cortex. Conversely, cortical neuroprogenitors labeled with the thymidine analog BrdU as a marker of active proliferation expressed lower levels of ATRX protein (Figure $1, \mathrm{I}-\mathrm{K})$. The elevated expression in postmitotic cells suggests a possible role for ATRX in the induction and/or maintenance of the differentiated state. To further define the role of ATRX in forebrain structures, we proceeded to eliminate ATRX expression by conditional mutagenesis in mice.

Conditional deletion of exon 18 is equivalent to a null mutation. Studies in yeast and Drosophila have demonstrated that inactivation of the ATPase activity of mating-type switching/SNF (SWI/SNF). The corresponding region in ATRX is encoded by exon 18, which allowed us to inactivate the full-length ATRX protein while leaving the shorter ATRXt isoform (exons 1-11) intact. A 6.2-kb Sst fragment spanning exons 17-20 of the Atrx gene was used to generate the targeting construct (Figure 2A) and, ultimately, Atr $x^{\text {floxed }}$ mice containing lox $\mathrm{P}$ sites flanking exon 18. Although these Atrx $x^{\text {floxed }}$ mice retained the neomycin-resistance gene $\left(n e o^{\mathrm{r}}\right)$, no ill effects on ATRX expression were observed (data not shown). When Atr $x^{\text {floxed }}$ mice were interbred with mice expressing transgenic Cre recombinase under the control of the ubiquitous GATA promoter, the consequent global deletion of Atrx caused early embryonic lethality, as described elsewhere (D. Garrick, W. Wood, A. Smith, R. Gibbons, and D. Higgs, unpublished observations). We attempted to circumvent early lethality by breeding the Atrx floxed mice to the Foxg1Cre mice previously characterized (29). Foxg1 expression is 

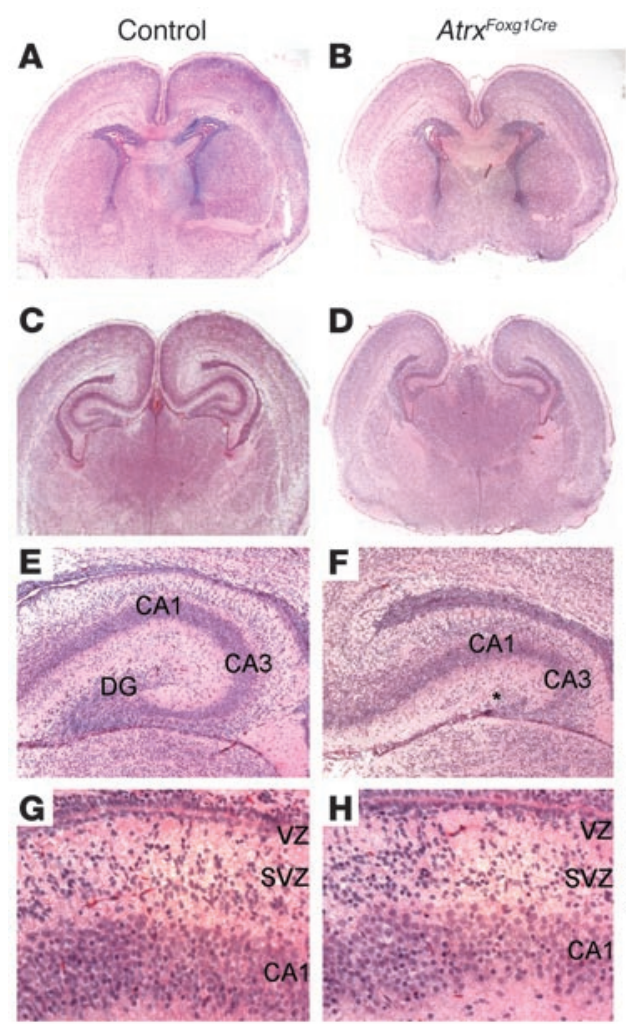

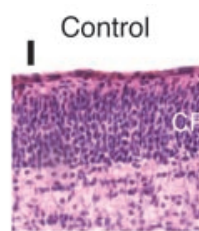

$\mathbf{K}$
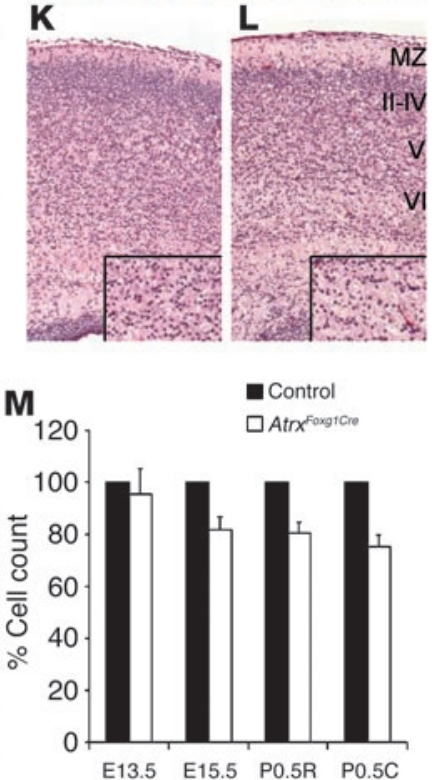

\section{Figure 4}

Cortical and hippocampal size reduction and hypocellularity. (A-L) Control (A, C, E, G, I, and K) and knockout (AtrxFoxg1Cre; B, D, F, H, J, and L) brain sections from P0.5 pups were stained with hematoxylin and eosin. Coronal views of rostral (A and $\mathbf{B})$ and caudal ( $\mathbf{C}$ and $\mathbf{D})$ forebrain demonstrate the size reduction of the cortex and hippocampus in the conditional knockout animals. There is dysgenesis of the hippocampus ( $E$ and $\mathbf{F}$ ) and lack of a dentate gyrus (asterisk in F) in Atrx Foxg1Cre males, as well as reduced cell density in the CA1 hippocampal field (G and $\mathbf{H})$. There is decreased cortical plate thickness in E15.5 AtrxFoxg1Cre cortex (I and $\mathbf{J})$. There is reduced cell density in rostral cortex at P0.5 in the Atrx Foxg1Cre cortex ( $K$ and $\mathbf{L}$ ). Insets show higher magnification. II-IV, V, and $\mathrm{VI}$ represent cortical layers present at birth. (M) Graph depicting cell numbers in the cortical plate at E13.5 and E15.5 and rostral (R) and caudal (C) sections at P0.5. Magnification, $\times 40(\mathbf{A}-\mathbf{L})$. first detected between E8 and E9 and is found in the telencephalic neuroepithelium, the basal ganglia, and the olfactory bulbs (34). Due to the location of Atrx on the X chromosome, the Cre-positive hemizygous male progeny resulting from this cross are Atrx null and will be referred to as Atrx ${ }^{\text {FoxglCre }}$ throughout this text.

Southern blot analysis of genomic DNA isolated from the cortex of Atrx ${ }^{\text {Foxg1Cre }}$ newborn pups demonstrated that Cre recombinase expression under the control of the Foxg1 promoter effectively deleted exon 18 (Figure 2B). RT-PCR analysis of cortical RNA isolated from mutant and wild-type animals confirmed the deletion of exon 18 (Figure 2C). Notably, the amount of this truncated RNA was greatly reduced, suggesting that it is unstable and probably results in reduced protein production. Indeed, immunoblot analyses of proteins isolated from the cortex demonstrated that the full-length ATRX protein was not detected with antibodies recognizing either the $\mathrm{N}$ - or C-terminal ends of the protein (Figure 2D; $39 \mathrm{f}$ and $\mathrm{H} 300$, respectively). Importantly, the knockout strategy did not generate any new ATRX isoforms that could provide a "gain-offunction" phenotype, yet the expression of the ATRXt isoform was maintained (Figure 2D, top panel).

To confirm the specificity of Cre expression resulting from the Foxg1Cre allele, we examined control and $\mathrm{Cr}^{+}$embryos for Cre transcripts by in situ hybridization (Figure 3, A and B). Cre expression in E13.5 embryos was detected at high levels in the developing forebrain, with some limited expression in the anterior retina (Figure 3, A and B). Cre expression was also observed later in development in the olfactory bulbs (data not shown). Cre activity was examined in the brains of newborn Atrx ${ }^{\text {Foxg1Cre }}$ pups (P0-P2) by immunohistochemistry (Figure 3, C-H). Cre recombinase was detected in the frontal cortex and hippocampus, whereas no expression was seen in control littermates (Figure 3, E and F). The expression of Cre always corre- lated with the lack of ATRX protein expression, while ATRX staining was still intense in other brain structures (Figure 3, C, D, and G). Nonetheless, there were a few cells within the forebrain in which no Cre activity was detected and ATRX expression persisted (Figure $3 \mathrm{H}$ ). Taken together, these results demonstrate that recombination of the Atr $x^{l o x \mathrm{P}}$ allele results in the loss of ATRX protein in the cortex and hippocampus of hemizygous male progeny.

Reduced weight and perinatal lethality in Atrx ${ }^{\text {Foxg1Cre offspring. The }}$ Atrx ${ }^{\text {Foxg1Cre }}$ conditional knockout males were smaller $(P<0.001)$ and did not suckle well, as evidenced by the lack of milk in their stomachs (Figure 3, I and J). These mice consequently died within the first 24-48 hours. Mendelian ratios at birth $(n=154)$ were close to the predicted value (25\%), with $23.4 \%$ Atr $x^{\text {Foxg1Cre }}$ males and $19.4 \%$ Atr $x^{\text {Foxg1Cre }}$ heterozygous females. Although this is not a statistically significant difference from the expected ratios, we often observed resorbed embryos (7\%) in litters from E11.5 to E18.5 that may represent animals in which Cre recombinase expression was widespread, as reported previously (29). Only 1 Atrx ${ }^{\text {Foxg1Cre }}$ male survived 24 days after birth. He was significantly smaller than the control littermates, became progressively weaker, and had to be sacrificed. A subset of heterozygous Atrx Foxg1Cre female mice also survived to adulthood and were fertile, but exhibited abnormal behavior such as nervousness and difficulty in rearing of pups.

Size reduction and bypocellularity in the cortex and hippocampus. In neonatal Atrx ${ }^{\text {Foxg1Cre }}$ mice, the frontal cortex was substantially reduced in size (Figure $3 \mathrm{~K}$ ), and histological analysis confirmed a reduction in cortical size that was greater in the caudal-medial cortex (Figure 4, A-D). Reduction of the subiculum and hippocampus was prominent, and in all Atrx ${ }^{\text {Foxg1Cre }}$ pups analyzed, the dentate gyrus was completely missing. In its place, a small mass of disorganized cells was observed (Figure 4; compare E with F). 

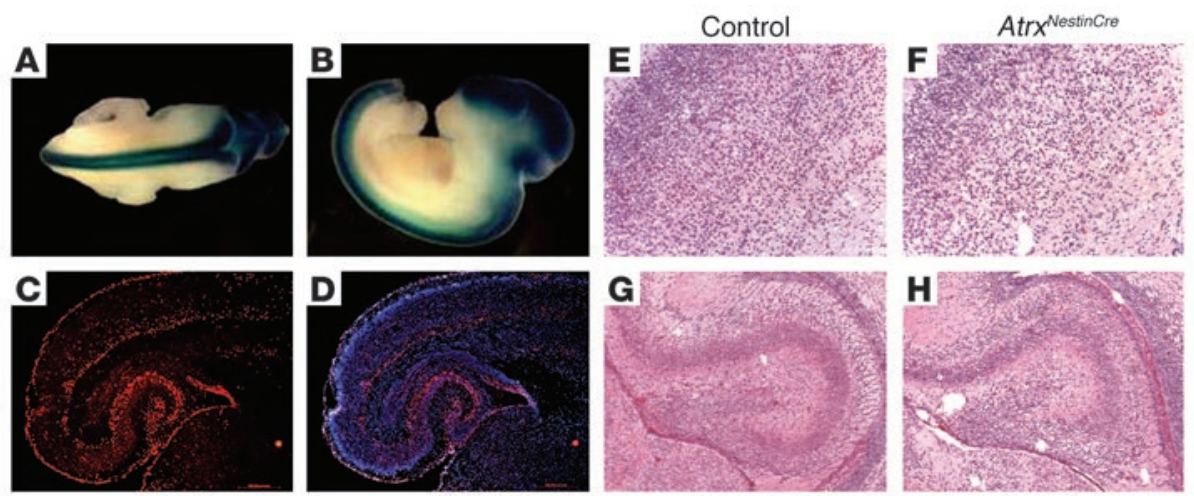

Figure 5

Atrx ${ }^{\text {NestinCre }}$ mice have a phenotype similar to that of AtrxFoxg1Cre animals. (A and B) In situ wholemount analysis of NestinCre-transgenic mice (E13.5) from line 2472 on a $\beta$-geo reporter strain background shows high Cre activity in the forebrain and spinal cord. Atrx ${ }^{\text {loxP }}$ mice were mated to NestinCre-transgenic mouse line 2472 to achieve selective recombination of the Atrx gene in neuronal

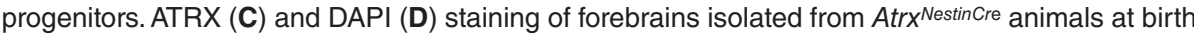
demonstrate ATRX expression in early-born neurons of the marginal zone and the subplate, plus variegated expression throughout the cortex and hippocampus. Hematoxylin and eosin staining of

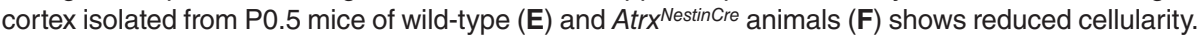
Similarly, neonatal hippocampal tissue sections from $\operatorname{Atrx}^{\text {NestinCre }}(\mathbf{H})$ and a control littermate $(\mathbf{G})$ also demonstrate hypocellularity in all hippocampal fields. Magnification, $\times 20(\mathbf{C}-\mathbf{H})$.

Closer examination of Atrx ${ }^{\text {FoxglCre }}$ cortical sections revealed a general decrease in cell density in the cortex. Cell counts in rostral and caudal sections showed that cell numbers were reduced by $20-30 \%$ in the cortical plate (Figure 4, K-M). We also observed a general reduction in hippocampal size (Figure $4 \mathrm{~F}$ ), with reduced numbers

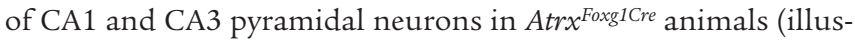
trated in the high magnification of the CA1 region in Figure 4H).

To establish the timing of cell loss during corticogenesis, we examined the cortex of embryos at several gestational time points. Although no reduction in cortical cell density was found at E13.5, statistically significant cell loss $(P<0.001)$ was present by $\mathrm{E} 15.5$, indicating a defect early in cortical development (Figure 4, I, J, and M).

Nextin promoter-driven Cre transgenic mice cause defects similar to those of Atrx Foxg1Cre animals. To confirm that Atrxx Foxg1Cre forebrain defects were attributable to the loss of ATRX protein expression, we generated transgenic animals that expressed Cre under the control of the Nestin promoter. Nestin is expressed in mice from E7.75 in the cerebellum and the ventricular and subventricular areas of the developing telencephalon, but not significantly in the hippocampal progenitors of dentate granule cells (35). We used the previously described NestinCre transgene (36) for pronuclear injection to generate several NestinCre-transgenic lines. Line 2472 showed strong expression in the forebrain and developing CNS

\section{Figure 6}

Many AtrxFoxg1Cre progenitor cells born at E15.5 never reach the superficial layers of the cortex and the dentate gyrus. Female mice homozygous for the Atrx floxed allele were bred to Foxg1Creheterozygous males and were injected with BrdU at E15.5, and newborn pups were sacrificed at P0.5. (A) Graph depicting BrdU staining of coronal sections of rostral and caudal forebrain. Fewer labeled cells migrate to the superficial layers of the cortex (rostral, $n=3, P<0.001$; caudal, $n=3, P<0.001$ ). Control was set as $100 \%$ labeling. (B and C) BrdU labeling in the dentate gyrus of cells at E15.5. Fewer labeled cells have migrated to the dentate gyrus in the ATRX-deficient hippocampus (C). Magnification, $\times 40$ (B and C). significant ATRX expression present in the neonatal hippocampus (Figure 5, C and D). Nonetheless, cell loss was consistently observed in the cortex of Atrx $x^{\text {NestinCre }}$ animals at P0.5, although to a lesser degree than in Atr $x^{\text {Foxg1Cre }}$ animals (Figure 5, E and F). The hippocampus was also affected to a lesser degree (Figure 5, G and H). Despite these slight differences, which we attribute to transgene differences, results with the Atrx ${ }^{\text {NestinCre }}$ mice confirm that hypocellularity of the developing forebrain is a specific effect of ATRX deficiency.

Fewer neurons migrate to superficial cortical layers and the dentate gyrus. To determine the reason for the cortical hypocellularity in Atrx ${ }^{\text {Foxg1Cre }}$ mice, we used neuronal "birthdating" experiments to monitor the fate of cells whose normal destination was the

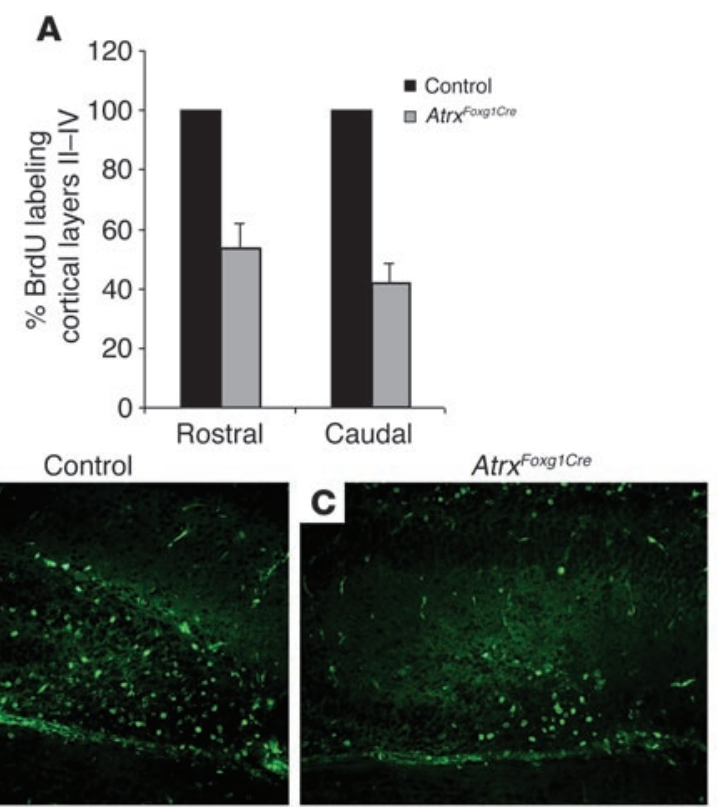



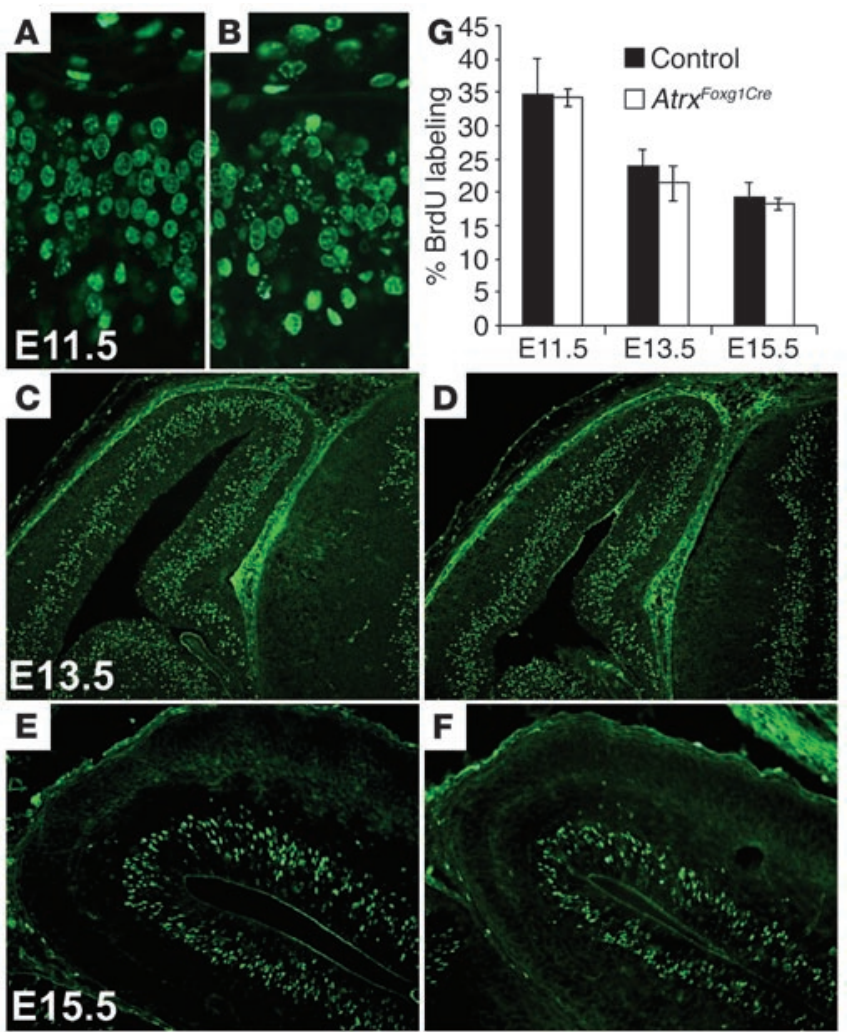

superficial layers of the cortex or the dentate gyrus. Pregnant mice were injected with BrdU to label dividing neural precursor cells at E15.5 and the pups were sacrificed at P0.5. The location of BrdU-positive cells in the cortex and hippocampus was examined by indirect immunofluorescence. In Atrx ${ }^{\text {Foxg1Cre }}$ pups, we observed that in both rostral and caudal regions of the forebrain, approximately 50\% fewer cells at E15.5 reached layers II-IV (rostral, $n=3$, $P<0.001$; caudal, $n=3, P<0.001)$ than did control cells that expressed high levels of ATRX protein (Figure 6A). This finding is in agreement with the hypocellularity of superficial layers observed in Atrx $x^{\text {Foxg1Cre }}$ animals at birth. Similarly, in the hippocampus, fewer dentate gyrus progenitors labeled with BrdU at E15.5 reached the dentate hilus than for normal littermates (Figure 6, B and C). Loss of BrdU-labeled cells could result from a decreased rate of progenitor cell proliferation, a defect in migration, or apoptotic death of neuroprogenitors before reaching their destination.

Embryonic neurogenesis is normal in Atrx ${ }^{\text {Foxg1Cre }}$ cortical progenitors. To examine whether cell loss in ATRX-deficient mutants resulted from decreased proliferation, we counted the replicating cortical

\section{Figure 8}

Increased number of pyknotic cell clusters during corticogenesis. (A-F) Cortical histology was examined at E11.5 (A and B) and E13.5 (C-F) by hematoxylin and eosin staining. Control littermates are depicted in $\mathbf{A}, \mathbf{C}$, and $\mathbf{D}$, and AtrxFoxg1Cre in B, E, and F. Large numbers of darkly stained pyknotic clusters (arrows) indicative of DNA condensation and apoptosis are observed in the cortex of E11.5 (B) and E13.5 (E and F) ATRX-deficient embryos. Greater numbers of these clusters are also present in the hippocampal area at E13.5 of AtrxFoxg1Cre embryos $(\mathbf{E}$ and $\mathbf{F})$ compared with controls (C and D). Magnification, $\times 40$ ( $\mathbf{A}$ and B), $\times 20$ (D and F), and $\times 10$ (C and E).

\section{Figure 7}

Normal proliferation in AtrxFoxg1Cre cortex during embryogenesis. Timed matings were set up between homozygous Atrx-floxed female mice and Foxg1Cre-heterozygous males. Pregnant female mice were subjected to a 1-hour BrdU pulse prior to sacrifice. Embryos were fixed and frozen, and sections were processed for BrdU immunostaining at E11.5 (A and B), E13.5 (C and D), E15.5 (E and F). Normal controls (A, C, and E) are compared with Atrx Foxg1Cre mice (B, D, and F). (G) BrdU-positive cells were counted in an identically sized area and data are expressed as a percentage of the total number of cells (DAPI-positive) at E11.5, E13.5, and E15.5. No statistically significant differences in proliferation were observed at any of the time points examined (E11.5, $n=4$, $P=1.000 ; \mathrm{E} 13.5, n=6, P=0.181 ; \mathrm{E} 15.5, n=5, P=0.482)$. Magnification, $\times 40(\mathbf{A}$ and $\mathbf{B}), \times 20(\mathbf{E}$ and $\mathbf{F})$, and $\times 10(\mathbf{C}$ and $\mathbf{D})$.

cells that were labeled after a 1-hour BrdU pulse. The fraction of cells in S phase during the BrdU pulse was determined at E11.5 (Figure 7, A and B; $n=4$ ), E13.5 (Figure 7, C and D; $n=6$ ), and $\mathrm{E} 15.5$ (Figure 7, E and F; $n=5$ ) from a minimum of 4 different wild-type and Atrx ${ }^{\text {Foxg1Cre }}$ littermate pairs. Despite the significant reduction in cortical size observed by E15.5, equivalent numbers of BrdU-positive progenitor cells were detected at each of these gestational time points (Figure 7G; E11.5, $P=1.000$; E13.5, $P=0.181$; E15.5, $P=0.482)$. Similarly, no change in the number of progenitors stained with the mitotic marker phosphohistone $\mathrm{H} 3$ was detected (data not shown). These data demonstrate that the loss of cortical cells during the development of Atrx ${ }^{\text {Foxg1Cre }}$ cortex is caused by a defect other than in proliferation.

Increased programmed cell death in the developing Atrx Foxg1Cre cortex. We rationalized that if neuronal progenitors proliferate normally, then cells must be lost as they undergo differentiation and start their radial migration toward the pial layer. In fact, the telencephalic cortex of E11.5 and E13.5 knockout embryos was distinguishable from that of normal controls by the presence of numerous clusters of pyknotic cells. These were easily identified by hematoxylin and eosin or DAPI staining and suggested that these cells were in late stages of apoptosis (Figure 8; compare A with B and $D$ with F). A striking number of these clusters were present in the hippocampal area at E13.5, with an average of $8.05 \pm 2.00$ clusters per section (SEM, 1.61; $n=6$ pairs) (Figure 8, E and F), while essentially none were found in normal littermates (Figure 8,
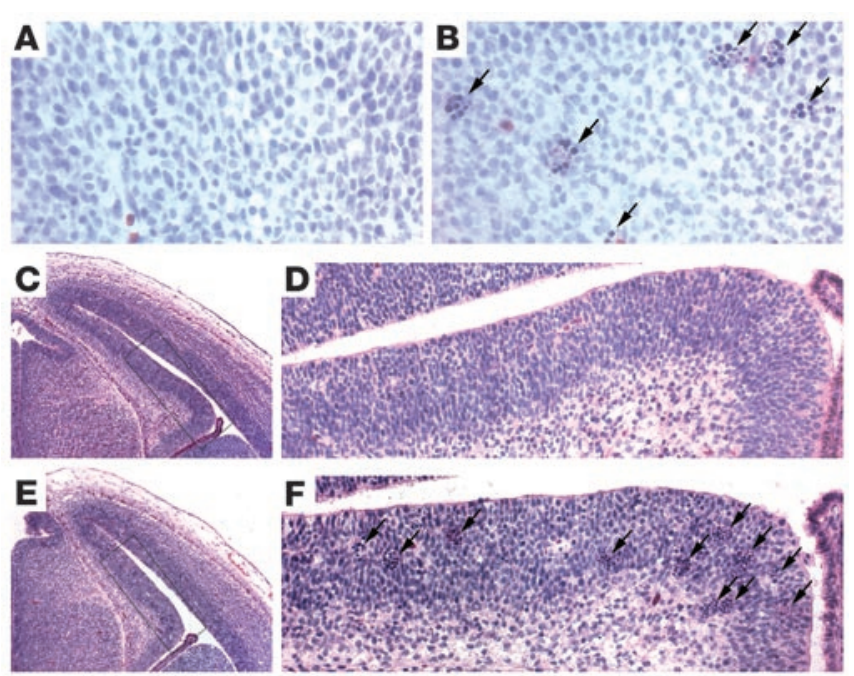


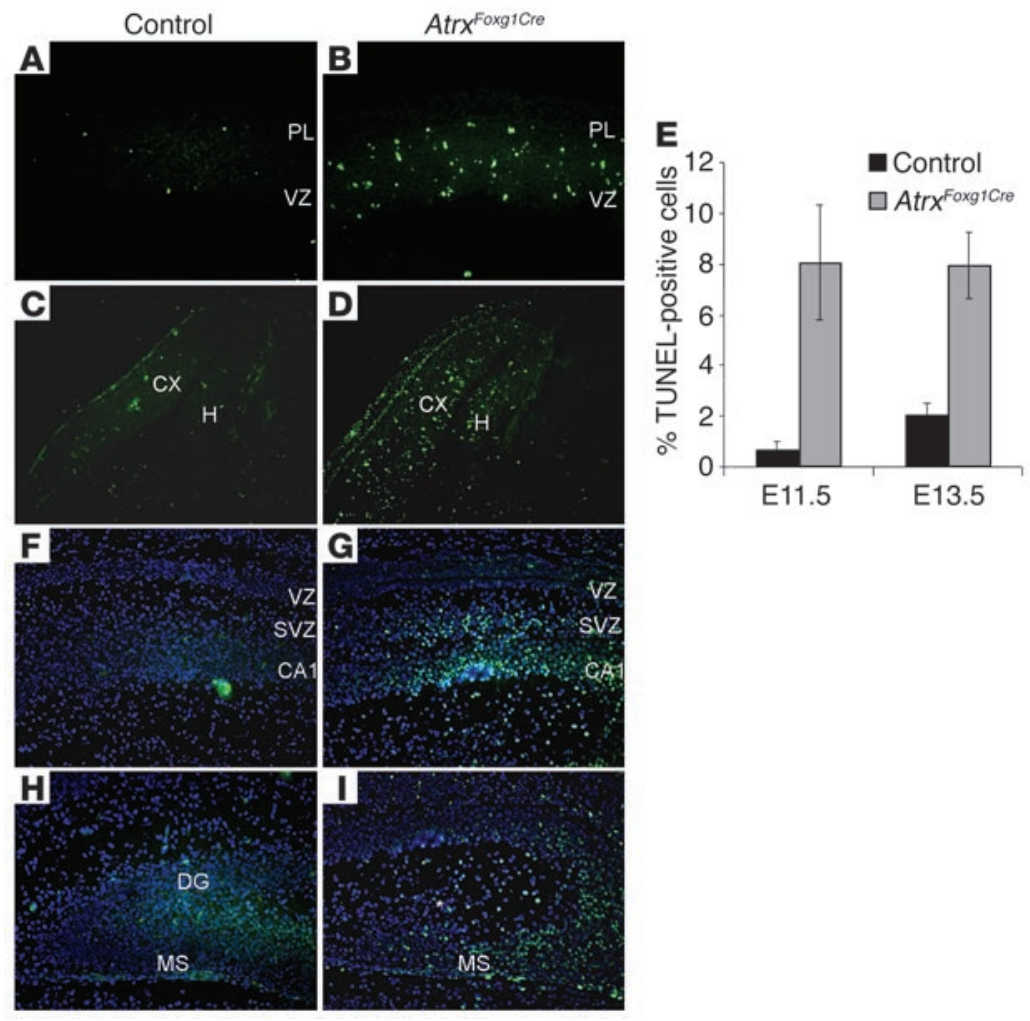

\section{Figure 9}

Increased apoptosis in AtrxFoxg1Cre embryonic cortex and postnatal hippocampus. (A-I) The TUNEL assay was performed on embryos at E11.5 (A and $\mathbf{B})$ and E13.5 (C and D) and at P0.5 in the hippocampus ( $\mathbf{H}$ and $\mathbf{I})$. Atry Foxg1Cre sections (B, D, G, and I) are compared with littermate controls ( $\mathbf{A}, \mathbf{C}, \mathbf{F}$, and $\mathbf{H})$. A substantial increase in the number of TUNEL-positive cells is observed in AtrxFoxg1Cre E11.5 neuroepithelial layer (compare A with $\mathbf{B}$ ). This increase persists in the cortex of E13.5 embryos ( $\mathbf{C}$ and $\mathbf{D}$ ) and is not restricted to the proliferative ventricular layer. (E) Graph depicting the increased number of apoptotic cells in ATRX-deficient embryos detected by TUNEL assay. TUNEL-positive cells were counted and data expressed as a percentage of total number of cells (DAPI-positive; E11.5, $n=3, P=0.045$; E13.5, $n=4$, $P<0.001$ ). At P0.5, increased levels of TUNEL-positive cells are detected in the subventricular zone of the CA1 field ( $\mathbf{F}$ and $\mathbf{G}$ ) and in the developing dentate gyrus (H and I). PL, pial layer; $\mathrm{H}$, hippocampus; MS, migratory stream. Missing DG in I is indicated by an asterisk. Magnification, $\times 20(\mathbf{A}, \mathbf{B}$, and $\mathbf{F}-\mathbf{I})$ and $\times 10(\mathbf{C}$ and $\mathbf{D})$.
C and D). We confirmed by TUNEL assay that there was a substantial increase in programmed cell death in ATRX-deficient embryos compared with that of control littermates. A 12-fold increased rate of cell death was observed at E11.5, when the neuroepithelium is comprised mainly of neuroprogenitors and a thin layer of differentiated cells of the preplate (Figure 9, A, B, and E; $n=3, P=0.045)$. We also observed a 4 -fold increase in TUNELpositive cells in ATRX-deficient embryos in the proliferative and differentiated cell layers at E13.5 (Figure 9, C-E; $n=4, P<0.001$ ). A reduction of cortical thickness was not apparent at this time during cortical development (Figure 8, C and E). At E15.5, a 2-fold increase in TUNEL staining was observed in Atrx ${ }^{\text {Foxg1Cre }}$ embryos, with no visible clusters of pyknotic cells. However, the cortical plate at E15.5 was thinner, with approximately $20 \%$ fewer cells in the mutant than in control embryos (Figure 4, I and J). We also

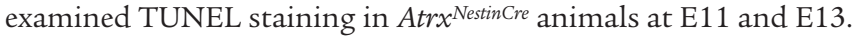
We observed TUNEL staining throughout these developmental stages; however, unlike the Atrx ${ }^{\text {Foxg1Cre }}$ embryos, there were no striking differences at the earlier developmental time points (data not shown). This difference may be attributable to the combination of the later onset and mosaic nature of transgene expression in the Atr $x^{\text {NestinCre }}$ mice. In newborn Atr $x^{\text {Foxg1Cre }}$ male pups, the number of cells undergoing apoptosis in the cortex as assessed by TUNEL staining was low and was not significantly different from that of controls (data not shown). However, cell death was detectable in the hippocampus, mainly in the subventricular zone of the CA1 field (Figure 9, F and G), in the dentate migratory stream, and in the few disorganized cells of the abnormal dentate gyrus (Figure 9, $\mathrm{H}$ and I). Taken together, the BrdU-labeling and TUNEL studies suggest that the loss of neurons in ATRX-deficient brains is caused by inappropriate apoptosis rather than defective proliferation.
Enhanced apoptosis is inherent to ATRX-deficient neuroprogenitors. The loss of ATRX in cortical and hippocampal progenitors is associated with excessive cell death upon differentiation, an outcome that is controlled either by an intrinsic signal or by environmental determinants of neuronal fate. To differentiate between these possibilities, we examined the consequence of ATRX loss in primary cortical progenitor cultures derived from E12.5 Atrx ${ }^{\text {Foxg1Cre }}$ embryos. These cell cultures have previously been shown to undergo the transition to postmitotic neurons in vitro (37). As described above, ATRX protein is expressed in control progenitors, but levels increase as the cells differentiate in culture. After 6 days in culture, many cells expressed neuronal differentiation markers, including MAP2, TUJ ( $\beta$-III tubulin), and NF200 (neurofilament protein, $200 \mathrm{kDa}$ ), demonstrating that ATRX-deficient progenitors are capable of differentiating and that ATRX expression is not essential for neuronal determination in vitro (Figure 10, C and D). Initially, we compared cell proliferation after 2 and 6 days in culture and observed no difference in BrdU labeling, as suggested by our earlier in vivo analysis (Figure 10, A and B). Although the level of apoptosis by TUNEL staining was low in both control and ATRX-deficient cells after 2 days in culture (data not shown), we observed increased numbers of apoptotic cells in ATRX-deficient progenitor cells after 6 days compared with that of the controls (Figure 10, E-H). This phenomenon was especially notable in cultured progenitors from heterozygous female embryos, in which only a subset of cells lacked ATRX protein due to the random pattern of X-inactivation. In mosaic progenitor cell colonies, the absence of ATRX protein always correlated with increased cell death, while the presence of the protein was protective and promoted survival (Figure 10I). These data demonstrate that ATRX protein is not required for the induction of some differentiation markers such as MAP2, but that in its absence, many 

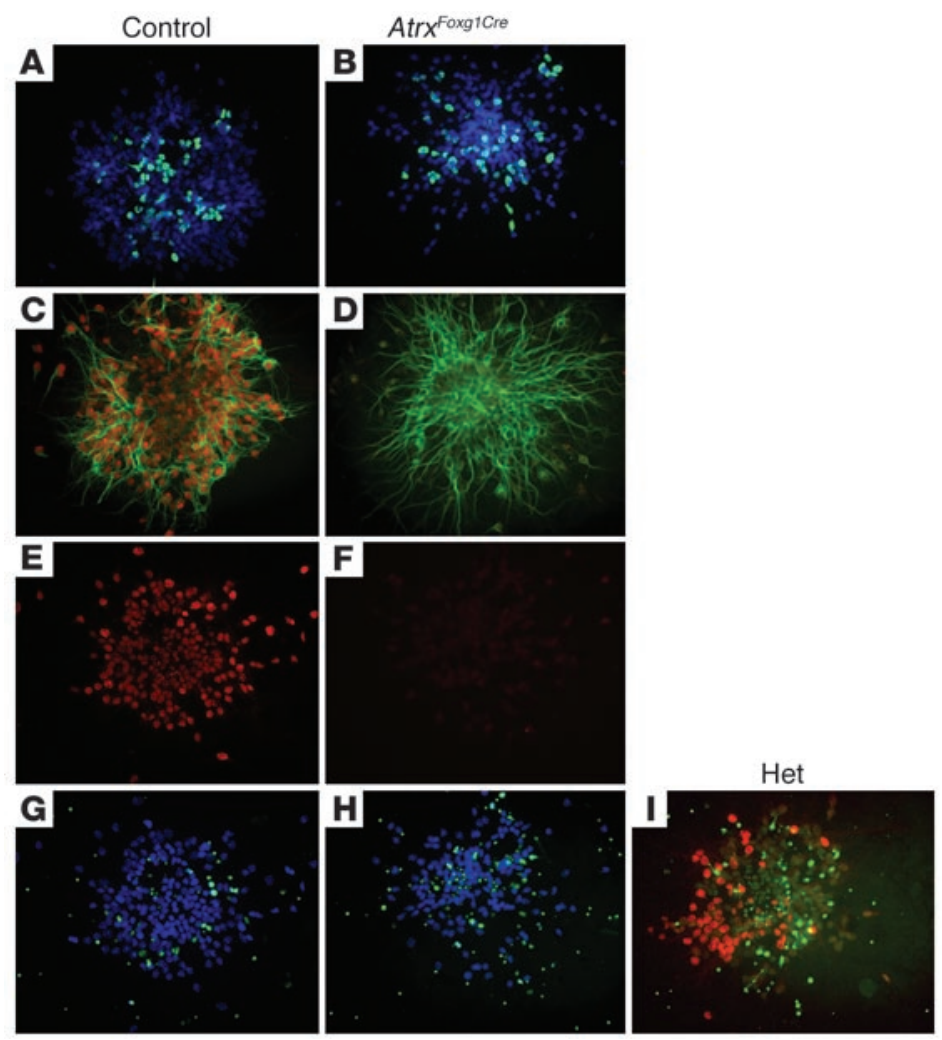

\section{Figure 10}

Increased apoptosis but normal proliferation in ATRX-deficient primary cortical cultures. (A-I) Primary cultures of cortical progenitor cells were established from E12.5 telencephalon of Atrx ${ }^{F o x g} 1 \mathrm{Cre}$ mice (B, D, $\mathbf{F}$, and $\mathbf{H}$ ), control Cre- littermates $(\mathbf{A}, \mathbf{C}, \mathbf{E}, \mathbf{G})$, or heterozygote (Het) Cre littermates (I), and were grown for 6 days in culture. (A and B) BrdU staining for 16 hours of control (A) and knockout (B) colonies demonstrates that the proliferative capacity of the cultured cells is not diminished in the absence of ATRX protein expression. (C and D) Control progenitors show a high level of ATRX protein (red) in differentiating MAP2-positive cells (green). Atrx Foxg1Cre progenitors that are ATRX deficient (lack of red staining in D) still express high levels of MAP2. (E and F) Corresponding ATRX staining of clones in $\mathbf{G}$ and $\mathbf{H}$ demonstrates the absence of ATRX

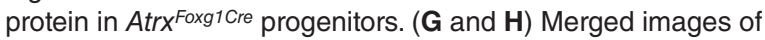
TUNEL-positive cells (green) and DAPI staining (blue). Note the increased level of TUNEL staining in Atrx ${ }^{\text {Foxg1Cre }}$ cell colonies after 6 days in culture. (I) Heterozygous $\mathrm{Cre}^{+}$progenitor cell clone stained for ATRX protein (red) and TUNEL (green), clearly showing that ATRX-deficient areas of the clone display higher levels of apoptosis. Magnification, $\times 20($ A-I). differentiating neurons undergo inappropriate cell death, a mechanism that is cell autonomous and inherent to neuroprogenitors.

\section{Discussion}

Programmed cell death plays an important role in cortical development by removing the excessive numbers of neurons that are generated in the subventricular zone but not incorporated into the cerebral layers (38-40). Utilizing 2 different Cre-transgenic matings to disrupt the murine Atrx gene, we observed significant and inappropriate levels of neuronal loss during corticogenesis. Specifically, this occurred during early stages (E11-E13.5) as well as postnatally in hippocampal progenitors and ex vivo in isolated progenitor cultures. Thus, the early onset of apoptosis in Atrxknockout animals demonstrates an important requirement for ATRX for survival during neuronal differentiation.

The idea of a role for ATRX in the developing neocortex is also supported by overexpression experiments in transgenic mice, as these animals generate excessive neuroprogenitors, culminating in a disorganized neuroepithelium layer and compromised embryonic survival (41). Certainly, gene regulation via chromatin remodeling at the time of neuronal differentiation is indicated, as genes driving cell cycle progression must be silenced while concurrently genes essential for differentiation require activation. Indeed, ablation of other transcription regulators such as the estrogen receptor $\beta$ and $\mathrm{Creb} / \mathrm{Crem}$ family members leads to hypocellularity due to inappropriate neuronal apoptosis $(42,43)$. Thus, the increased apoptosis could result from a large number of direct or indirect mechanisms. These mechanisms include a failure to induce expression of neurotrophic factors or their receptors, altered expression of genes involved in the apoptotic cascade, or the inappropriate regulation of genes that ultimately trigger a cell death pathway. However, ATRX is not essential for neuronal survival, as about $75 \%$ of cells survive. Thus, the stochastic frequency of apoptosis in Atrx ${ }^{\text {Foxg1Cre }}$ mice suggests that compensating factors can overcome the functional loss of ATRX protein in most cells. Alternatively, the loss of ATRX may generate a hypersensitive state such that the cells are more apt to "choose" death over survival during the stress that accompanies differentiation and cell cycle withdrawal.

Recently, ATRX was shown to associate in a complex with DAXX $(24,25)$, a protein with both transcriptional repressive and proapoptotic functions that localizes to PML nuclear bodies. Extensive studies have demonstrated that both DAXX and PML are regulators of apoptosis. DAXX was originally shown to control Fas-mediated apoptosis through activation of ASK1 and induction of the Jun $\mathrm{N}$-terminal kinase pathway $(44,45)$. Similarly, it is well documented that PML plays a role in the activation of multiple p53-dependent and -independent (Fas- and TNF-induced) apoptotic pathways (46). PML and DAXX cooperate in potentiating Fas induced apoptosis, probably by modulating the transcription of genes involved in Fasinduced caspase activation and apoptosis (47). Because ATRX and DAXX interact, one may speculate that loss of ATRX may compromise the regulation of apoptotic genes increasing the sensitivity of the cell to proapoptotic signals such as stress.

Dysregulation of programmed cell death in the neuroprogenitor population is predicted to manifest as microcephaly and cognitive delay. Studies of Down syndrome and the corresponding trisomy 16 mouse model have reported large increases in cell death with a significant reduction in cortical mass (48-52). Similar parallels can be drawn between the ATRX-deficient mice and the human disorder. Microcephaly has been reported in a large majority (76\%) of ATR-X patients (9). In addition, the Atrx ${ }^{\text {Foxg1Cre }}$ mice presented with severe hypotonia and feeding difficulties at birth, which is a common symptom observed in about $85 \%$ of ATR-X patients (9). Indeed, 
the hippocampal dysgenesis in ATRX-deficient mice could provide a ready explanation for the neuronal deficiencies, such as severe learning and memory deficits and a high incidence of seizures (35-40\%), that are present in affected males (9). Nevertheless, ATRX-deficient mice rarely survive past the first 2 postnatal days. This rather severely deleterious phenotype is probably a consequence of the complete loss of ATRX in this mouse model. At present there are no human mutations that are known to be null. Some premature stop mutations, which were previously thought to be null, produce full-length protein (R.J. Gibbons et al., unpublished observations) and SNF2 domain missense mutations attenuate ATPase activity (25).

In conclusion, we have demonstrated here that loss of ATRX function within the forebrain significantly affects normal corticogenesis. Moreover, our data suggest that ATRX can play a role in normal cell differentiation in general, and this may contribute to many of the known features of the ATR-X syndrome.

\section{Methods}

Generation of mice. A 6.2-kb SstI fragment containing exons 17-20 of the murine Atrx gene was isolated from a 129-derived genomic library and was subcloned into pCR-ScriptSK ${ }^{+}$(Stratagene) from which the BamHI site had previously been removed. Using oligonucleotide linkers, a unique Eco47III site midway between exons 17 and 18 was converted to a BamHI site for the insertion of the Bam HI fragment comprising a neomycin-selectable marker flanked by loxP sites (MC1neopA). A BglII site was introduced by mutagenesis into the middle of intron 18 to allow insertion of additional loxP and SstI sites. An XhoI-SalI fragment, MC1tk dimer, containing 2 copies of the herpes simplex virus thymidine kinase gene driven by the F9 enhancer, was inserted in the SalI cloning site of the vector. This construct was linearized with XhoI prior to electroporation. Recombinants were screened by Southern blot with probes flanking the homology arms. Animals were generated by blastocyst injection in collaboration with A. Smith (University of Edinburgh, Edinburgh, United Kingdom).

The Foxg1Cre transgenic line (29) was kindly provided by S. McConnell (Stanford University, Stanford, California, USA). NestinCre mice were generated by pronuclear injection of the nestin-Cre plasmid (generously provided by P.C. Orban, University of British Columbia, Vancouver, British Columbia, Canada) into mouse embryos from $\mathrm{FVB} / \mathrm{N}$ mice as described previously (41). Transgenic mice were analyzed for recombination in situ using reporter mice carrying a $\beta$-galactosidase transgene whose expression was dependent on Cre-mediated recombination. All animal studies were approved by the University of Ottawa Animal Care Committee, accredited by the Canadian Council on Animal Care.

Animal husbandry/genotyping. Mice conditionally deficient in ATRX were generated by crossing of Atrx ${ }^{\text {loxP }}$ females (C57BL6 background) with heterozygous Foxg1Cre-knock-in male mice (FVB/N; ref. 29) or with NestinCre-heterozygous males (FVB/N background). DNA from tail tissue of newborn pups or yolk sac DNA from embryos was genotyped by PCR with the following primers: $17 \mathrm{~F}, 18 \mathrm{R}$, and $n e o^{\mathrm{r}}$. For Southern blot analysis, a DNA fragment spanning exons 17 and 18 was used as a probe and hybridized to mouse genomic DNA digested with SstI. For developmental studies, midday of the day of vaginal plug discovery was considered E0.5. At scheduled times, pregnant females were anesthetized by $\mathrm{CO}_{2}$ and sacrificed by cervical dislocation.

RT-PCR. Five micrograms of total RNA obtained from Atrx ${ }^{\text {Foxglcre }}$ and littermate control cortices was reverse-transcribed as described (41) and was used for PCR amplification with the following primers: Mxnp31 (exon 18)/Mxnp30 (exon 20), 17F (exon17)/Mxnp30 (exon 20), and GAPDHF/ GAPDHR as control primers. Conditions for amplification were as follows: $95^{\circ} \mathrm{C}$ for 3 minutes, followed by 30 cycles of $95^{\circ} \mathrm{C}$ for 30 seconds, $55^{\circ} \mathrm{C}$ for 30 seconds, and $72^{\circ} \mathrm{C}$ for 10 minutes.
Histology and immunofluorescence. Embryos and postnatal brains were fixed and sectioned as described previously (41). For histology, slides were stained with hematoxylin and eosin. For immunofluorescence staining, sections were incubated with the primary antibody overnight at $4{ }^{\circ} \mathrm{C}$, washed for 1 hour in PBS, and incubated with the secondary antibody for 1 hour. Sections were counterstained with DAPI and mounted in Vectashield (Vector Laboratories, Inc). The following primary antibodies were used: anti-ATRX, rabbit polyclonal (1:100 dilution; Santa Cruz Biotechnology Inc.) anti-Cre recombinase, mouse monoclonal (1:100 dilution; Babco), anti-TuJ1 (mouse monoclonal hybridoma supernatant,1:25 dilution; from D. Brown, University of Ottawa, Ottawa, Ontatio, Canada) anti-phosphohistone H3 (1:100 dilution), anti-MAP2 mouse monoclonal (1:100 dilution; Sigma-Aldrich), and anti-NF200, rabbit polyclonal (1:100 dilution; Sigma-Aldrich). The secondary antibodies used were goat anti-rabbitAlexa 594 (1:2,000 dilution; Molecular Probes) and goat anti-mouse-FITC (1:100 dilution; Sigma-Aldrich). The TUNEL assay was performed using the In Situ Cell Death Detection Kit (Roche Diagnostics) according to the manufacturer's instructions.

BrdU labeling. For labeling of cells in S phase, homozygous Atrx $x^{\text {lox } \mathrm{P}}$ mice obtained from timed matings were injected intraperitoneally with $14 \mathrm{mg}$ BrdU (Sigma-Aldrich) per $100 \mathrm{~g}$ body weight (53). Animals were sacrificed after 1 hour by cervical dislocation and the embryos were recovered in icecold PBS, pH 7.4, and were fixed in $4 \%$ paraformaldehyde. For birthdating experiments, BrdU was injected intraperitoneally into pregnant mice at $\mathrm{E} 11.5$, E13.5, and E15.5, and brains were dissected from neonatal offspring (P0.5). Incorporation of modified nucleotide was detected by staining with anti-BrdU (BU-20) as described previously (54).

Primary neuroprogenitor cultures. Cortical progenitor cell cultures were prepared as described previously $(55,56)$ using cortices dissected from E12.5 embryos. Cells were processed for immunofluorescence and TUNEL staining 2 and 6 days after plating. For BrdU-labeling experiments, cells were incubated for 16 hours in the presence of $10 \mu \mathrm{M} \mathrm{BrdU}$ and were fixed for 10 minutes with cold $\mathrm{EtOH}: \mathrm{MeOH}$ (3:1). Cells were pretreated for 20 minutes with $2 \mathrm{~N} \mathrm{HCl}$ to denature DNA and for 10 minutes with $0.1 \mathrm{M} \mathrm{Na}_{2} \mathrm{~B}_{4} \mathrm{O}_{7}, \mathrm{pH}$ 8.5. Cells were washed 3 times with PBS and were incubated for 1 hour with a 1:100 dilution of primary anti-BrdU (BU-20) in $2 \%$ BSA/PBS. After 3 washes with PBS, cells were incubated for 1 hour with secondary anti-mouse-FITC (1:100 dilution) in $2 \%$ BSA/PBS and were counterstained with DAPI.

\section{Acknowledgments}

We thank Maribeth Lazzaro, Rashmi Kothary, and Valerie Wallace for review of the manuscript. We also thank Tiffany Bokor and Rebecca Walsh for technical support. This work was supported by a grant from the Canadian Institutes of Health Research (CIHR) (MOP-66970) to D.J. Picketts. N.G. Bérubé was supported by a CIHR postdoctoral fellowship and D.J. Picketts is a CIHR New Investigator.

Received for publication June 2, 2004, and accepted in revised form November 30, 2004.

Address correspondence to: David J. Picketts, Ottawa Health Research Institute, Molecular Medicine Program, 501 Smyth Road, Ottawa, Ontario K1H8L6, Canada. Phone: (613) 737-8989; Fax: (613) 737-8803; E-mail: dpicketts@ohri.ca.

Nathalie G. Bérubé's present address is: Departments of Biochemistry and Paediatrics, University of Western Ontario, Lawson Health Research Institute, and Human Molecular Genetics Group, Child Health Research Institute, London, Ontario, Canada. 
1. Gupta, A., Tsai, L.H., and Wynshaw-Boris, A. 2002. Life is a journey: a genetic look at neocortical development. Nat. Rev. Genet. 3:342-355.

2. Gotz, M., Stoykova, A., and Gruss, P. 1998. Pax6 controls radial glia differentiation in the cerebral cortex. Neuron. 21:1031-1044.

3. Pellegrini, M., Mansouri, A., Simeone, A., Boncinelli, E., and Gruss, P. 1996. Dentate gyrus formation requires Emx2. Development. 122:3893-3898.

4. Zhou, C., Tsai, S.Y., and Tsai, M.J. 2001. COUP-TFI: an intrinsic factor for early regionalization of the neocortex. Genes Dev. 15:2054-2059.

5. Chelly, J., and Mandel, J.L. 2001. Monogenic causes of X-linked mental retardation. Nat. Rev. Genet 2:669-680.

6. Gibbons, R.J., Picketts, D.J., Villard, L., and Higgs, D.R. 1995. Mutations in a putative global transcriptional regulator cause X-linked mental retardation with alpha-thalassemia (ATR-X syndrome). Cell. 80:837-845.

7. Trivier, E., et al. 1996. Mutations in the kinase Rsk2 associated with Coffin-Lowry syndrome. Nature. 384:567-570.

8. Amir, R.E., et al. 1999. Rett syndrome is caused by mutations in X-linked MECP2, encoding methylCpG-binding protein 2. Nat. Genet. 23:185-188.

9. Gibbons, R.J., and Higgs, D.R. 2000. Molecularclinical spectrum of the ATR-X syndrome. Am. J. Med. Genet. 97:204-212.

10. Gibbons, R.J., et al. 1997. Mutations in transcriptional regulator ATRX establish the functional significance of a PHD-like domain. Nat. Genet. 17:146-148.

11. Villard, L., et al. 1996. XNP mutation in a large family with Juberg-Marsidi syndrome. Nat. Genet. 12:359-360.

12. Abidi, F., et al. 1999. Carpenter-Waziri syndrome results from a mutation in XNP. Am. J. Med. Genet. 85:249-251.

13. Villard, L., Fontes, M., Ades, L.C., and Gecz, J. 2000. Identification of a mutation in the XNP/ATR-X gene in a family reported as Smith-Fineman-Myers syndrome. Am. J. Med. Genet. 91:83-85.

14. Lossi, A.M., et al. 1999. Mutation of the XNP/ ATR- $X$ gene in a family with severe mental retardation, spastic paraplegia and skewed pattern of $\mathrm{X}$ inactivation: demonstration that the mutation is involved in the inactivation bias. Am. J. Hum. Genet. 65:558-562.

15. Yntema, H.G., et al. 2002. Expanding phenotype of XNP mutations: mild to moderate mental retardation. Am. J. Med. Genet. 110:243-247.

16. Guerrini, R., et al. 2000. A nonsense mutation of the ATRX gene causing mild mental retardation and epilepsy. Ann. Neurol. 47:117-121.

17. Aasland, R., Gibson, T.J., and Stewart, A.F. 1995. The PHD finger: implications for chromatin-mediated transcriptional regulation. Trends Biochem. Sci. 20:56-59.

18. Eisen, J.A., Sweder, K.S., and Hanawalt, P.C. 1995. Evolution of the SNF2 family of proteins: subfamilies with distinct sequences and functions. Nucleic Acids Res. 23:2715-2723.

19. Picketts, D.J., Tastan, A.O., Higgs, D.R., and Gibbons, R.J. 1998. Comparison of the human and murine ATRX gene identifies highly conserved, functionally important domains. Mamm. Genome. 9:400-403.

20. Picketts, D.J., et al. 1996. ATRX encodes a novel member of the SNF2 family of proteins: mutations point to a common mechanism underlying the ATR-X syndrome. Hum. Mol. Genet. 5:1899-1907.

21. Garrick, D., et al. 2004. A conserved truncated isoform of the ATR-X syndrome protein lacking the SWI/SNF-homology domain. Gene. 326:23-34.

22. Berube, N.G., Smeenk, C.A., and Picketts, D.J. 2000. Cell cycle-dependent phosphorylation of the ATRX protein correlates with changes in nuclear matrix and chromatin association. Hum. Mol. Genet. 9:539-547.

23. McDowell, T.L., et al. 1999. Localization of a putative transcriptional regulator (ATRX) at pericentromeric heterochromatin and the short arms of acrocentric chromosomes. Proc. Natl. Acad. Sci. U. S. A. 96:13983-13988.

24. Xue, Y., et al. 2003. The ATRX syndrome protein forms a chromatin-remodeling complex with Daxx and localizes in promyelocytic leukemia nuclear bodies. Proc. Natl. Acad. Sci. U. S. A. 100:10635-10640.

25. Tang, J., et al. 2004. A novel transcription regulatory complex containing Daxx and the ATR-X syndrome protein. J. Biol. Chem. 279:20369-20377.

26. Bachman, K.E., Rountree, M.R., and Baylin, S.B. 2001. Dnmt3a and Dnmt $3 b$ are transcriptional repressors that exhibit unique localization properties to heterochromatin. J. Biol. Chem. 276:32282-32287.

27. Gibbons, R.J., et al. 2000. Mutations in ATRX, encoding a SWI/SNF-like protein, cause diverse changes in the pattern of DNA methylation. Nat. Genet. 24:368-371.

28. Gibbons, R.J., et al. 2003. Identification of acquired somatic mutations in the gene encoding chromatin-remodeling factor ATRX in the alpha-thalassemia myelodysplasia syndrome (ATMDS). Nat. Genet. 34:446-449.

29. Hebert, J.M., and McConnell, S.K. 2000. Targeting of cre to the Foxg1 (BF-1) locus mediates loxP recombination in the telencephalon and other developing head structures. Dev. Biol. 222:296-306.

30. Stayton, C.L., et al. 1994. Cloning and characterization of a new human Xq13 gene, encoding a putative helicase. Hum. Mol. Genet. 3:1957-1964.

31. Cote, J., Quinn, J., Workman, J.L., and Peterson, C.L. 1994. Stimulation of GAL4 derivative binding to nucleosomal DNA by the yeast SWI/SNF complex. Science. 265:53-60.

32. Khavari, P.A., Peterson, C.L., Tamkun, J.W., Mendel, D.B., and Crabtree, G.R. 1993. BRG1 contains a conserved domain of the SWI2/SNF2 family necessary for normal mitotic growth and transcription. Nature. 366:170-174.

33. Elfring, L.K., et al. 1998. Genetic analysis of brahma: the Drosophila homolog of the yeast chromatin remodeling factor SWI2/SNF2. Genetics. 148:251-265.

34. Dou, C.L., Li, S., and Lai, E. 1999. Dual role of brain factor- 1 in regulating growth and patterning of the cerebral hemispheres. Cereb. Cortex. 9:543-550.

35. Dahlstrand, J., Lardelli, M., and Lendahl, U. 1995. Nestin mRNA expression correlates with the central nervous system progenitor cell state in many, but not all, regions of developing central nervous system. Brain Res. Dev. Brain Res. 84:109-129.

36. Tronche, F., et al. 1999. Disruption of the glucocorticoid receptor gene in the nervous system results in reduced anxiety. Nat. Genet. 23:99-103.

37. Ghosh, A., and Greenberg, M.E. 1995. Distinct roles for bFGF and NT-3 in the regulation of cortical neurogenesis. Neuron. 15:89-103.

38. Blaschke, A.J., Staley, K., and Chun, J. 1996. Widespread programmed cell death in proliferative and postmitotic regions of the fetal cerebral cortex. Development. 122:1165-1174.

39. Pettmann, B., and Henderson, C.E. 1998. Neuronal cell death. Neuron. 20:633-647.

40. Yuan, J., and Yankner, B.A. 2000. Apoptosis in the nervous system. Nature. 407:802-809.

41. Berube, N.G., et al. 2002. Neurodevelopmental defects resulting from ATRX overexpression in transgenic mice. Hum. Mol. Genet. 11:253-261.

42. Wang, L., Andersson, S., Warner, M., and Gustafsson, J.A. 2003. Estrogen receptor (ER)beta knockout mice reveal a role for ERbeta in migration of cortical neurons in the developing brain. Proc. Natl. Acad. Sci. U. S. A. 100:703-708.

43. Mantamadiotis, T., et al. 2002. Disruption of CREB function in brain leads to neurodegeneration. Nat. Genet. 31:47-54.

44. Chang, H.Y., Nishitoh, H., Yang, X., Ichijo, H., and Baltimore, D. 1998. Activation of apoptosis signalregulating kinase 1 (ASK1) by the adapter protein Daxx. Science. 281:1860-1863.

45. Yang, X., Khosravi-Far, R., Chang, H.Y., and Baltimore, D. 1997. Daxx, a novel Fas-binding protein that activates JNK and apoptosis. Cell. 89:1067-1076.

46. Bernardi, R., and Pandolfi, P.P. 2003. Role of PML and the PML-nuclear body in the control of programmed cell death. Oncogene. 22:9048-9057.

47. Torii, S., Egan, D.A., Evans, R.A., and Reed, J.C. 1999. Human Daxx regulates Fas-induced apoptosis from nuclear PML oncogenic domains (PODs). EMBO J. 18:6037-6049.

48. Kadota, M., Shirayoshi, Y., and Oshimura, M. 2002. Elevated apoptosis in pre-mature neurons differentiated from mouse ES cells containing a single human chromosome 21. Biochem. Biophys. Res. Commun. 299:599-605.

49. Seidl, R, et al. 2001. Evidence for apoptosis in the fetal Down syndrome brain. J. Child Neurol. 16:438-442.

50. Haydar, T.F., Nowakowski, R.S., Yarowsky, P.J., and Krueger, B.K. 2000. Role of founder cell deficit and delayed neuronogenesis in microencephaly of the trisomy 16 mouse. J. Neurosci. 20:4156-4164.

51. Nagy, Z. 1999. Mechanisms of neuronal death in Down's syndrome. J. Neural Transm. Suppl. 57:233-245.

52. Bambrick, L.L., and Krueger, B.K. 1999. Neuronal apoptosis in mouse trisomy 16: mediation by caspases. J. Neurochem. 72:1769-1772.

53. Miller, M.W., and Nowakowski, R.S. 1988. Use of bromodeoxyuridine-immunohistochemistry to examine the proliferation, migration and time of origin of cells in the central nervous system. Brain. Res. 457:44-52.

54. Magaud, J.P., et al. 1989. Double immunocytochemical labeling of cell and tissue samples with monoclonal anti-bromodeoxyuridine. J. Histochem. Cytochem. 37:1517-1527.

55. Gloster, A., El-Bizri, H., Bamji, S.X., Rogers, D., and Miller, F.D. 1999. Early induction of Talpha1 alphatubulin transcription in neurons of the developing nervous system. J. Comp. Neurol. 405:45-60.

56. Slack, R.S., El-Bizri, H., Wong, J., Belliveau, D.J., and Miller, F.D. 1998. A critical temporal requirement for the retinoblastoma protein family during neuronal determination. J. Cell Biol. 140:1497-1509. 